\title{
SPECIAL LAGRANGIAN SUBMANIFOLDS OF THE NEARLY KAEHLER 6-SPHERE
}

\author{
LUC VRANCKEN \\ LAMATH, ISTV2, Université de Valenciennes, Le Mont Houy, \\ 59313 Valenciennes cedex 9, France \\ e-mail:luc.vrancken@univ-valenciennes.fr
}

(Received 23 January, 2002; accepted 26 March, 2002)

\begin{abstract}
In this paper, we study Lagrangian submanifolds $M$ of the nearly Kähler 6-sphere $S^{6}(1)$. It is well known that such submanifolds, which are 3dimensional, are always minimal and admit a symmetric cubic form. Following an idea of Bryant, developed in the study of Lagrangian submanifolds of $\mathbb{C}^{3}$, we then investigate those Lagrangian submanifolds for which at each point the tangent space admits an isometry preserving this cubic form. We obtain that all such Lagrangian submanifolds can be obtained starting from complex curves in $S^{6}(1)$ or from holomorphic curves in $\mathbb{C} P^{2}(4)$. In the final section we classify the Lagrangian submanifolds which admit a Sasakian structure that is compatible with the induced metric. This last result generalizes theorems obtained by Deshmukh and ElHadi. Note that in this case, the condition that $M$ admits a Sasakian structure implies that $M$ admits a pointwise isometry of the tangent space.
\end{abstract}

2000 Mathematics Subject Classification. 53B25, 53D12.

1. Introduction. It is well known that starting from the Cayley numbers, it is possible to introduce an almost complex structure $J$ on the 6-dimensional sphere $S^{6}(1)$ which is compatible with the standard metric. It was shown by Calabi and Gluck, see [5] that this structure, from a geometric viewpoint, is the best possible almost complex structure on $S^{6}(1)$. In the study of submanifolds, it is then natural to study submanifolds for which $J$ preserves the tangent space (and hence also the normal space) and those for which $J$ interchanges the tangent and normal spaces. The first class are called almost complex submanifolds and it was shown by Gray that they have to be two dimensional (complex one dimensional). Further results about these complex curves were obtained amongst others in [4], [10] and [2].

The second class of submanifolds mentioned, which by its definition have to be 3-dimensional, are called Lagrangian submanifolds. They were first investigated by Ejiri, [13], who showed that a Lagrangian submanifold is always orientable and minimal. Moreover, as is the case for Lagrangian submanifolds of complex space forms, we have that the 3-form $C$ defined by

$$
C(X, Y, Z)=\langle h(X, Y), J Z\rangle,
$$

where $h$ denotes the second fundamental form of the immersion is always symmetric. This implies that at every point $p$ of $M$, the cubic form can be represented by a 
homogeneous polynomial $f_{p}$ of degree 3 given by

$$
f_{p}(x, y, z)=\left\langle h\left(x e_{1}+y e_{2}+z e_{3}, x e_{1}+y e_{2}+z e_{3}\right), J\left(x e_{1}+y e_{2}+z e_{3}\right)\right\rangle,
$$

where $\left\{e_{1}, e_{2}, e_{3}\right\}$ is an orthonormal basis at the point $p$. As $M$ is minimal we see that the trace of this cubic form with respect to the metric vanishes. As far as such homogeneous polynomials of degree 3 with vanishing trace on a 3-dimensional real vector space are concerned, we quote the following result by Bryant [3].

THEOREM 1. Let $p \in M$ and assume that there exist an orientation preserving isometry of the tangent space which preserves $f_{p}$. Then there exists an orthonormal basis of $T_{p} M$ such that either

(i) $f_{p}=0$, in this case $f_{p}$ is preserved by every isometry,

(ii) $f_{p}=\lambda\left(2 x^{3}-3 x y^{2}-3 x z^{2}\right)$, for some positive number $\lambda$ in which case $f_{p}$ is preserved by a 1-parameter group of rotations,

(iii) $f_{p}=6 \lambda x y z$ for some positive number $\lambda$, in which case $f_{p}$ is preserved by the discrete group $A_{4}$ of order 12 ,

(iv) $f_{p}=\lambda\left(x^{3}-3 x y^{2}\right)$ for some positive number $\lambda$, in which case $f_{p}$ is preserved by the discrete group $S_{3}$ of order 6 ,

(v) $f_{p}=\lambda\left(2 x^{3}-3 x y^{2}-3 x z^{2}\right)+\mu\left(y^{3}-3 x y^{2}\right)$ for some $\lambda, \mu>0$, with $\mu \neq \sqrt{2} \lambda$, in which case $f_{p}$ is preserved by the group $\mathbb{Z}_{3}$,

(vi) $f_{p}=\lambda\left(2 x^{3}-3 x y^{2}-3 x z^{2}\right)+6 \mu x y z$, for some $\lambda, \mu>0$, with $\lambda \neq \mu$, in which case $f_{p}$ is preserved by the group $\mathbb{Z}_{2}$ of order 2 .

In Section 3, we will assume that one of the special cases of the above theorem is satisfied at every point of the Lagrangian submanifold. We call $M$ a Lagrangian submanifold of Type (k) if and only if Theorem $1(\mathrm{k})$ is satisfied at every point $p$ of $M$. As it turns out, several of these classes of Lagrangian submanifolds have been previously studied. It follows easily that for Lagrangian submanifolds of Type (i) up to (v) can be characterised by the fact that the Ricci tensor admits at least a double eigenvalue. Therefore they can be easily recovered from the classification results in [13] (Type (i) and Type (iii)), [11] (Type (iv)) and [9] (Type (ii) and Type (v)). All such Lagrangian submanifolds can be obtained starting from complex curves in $S^{6}(1)$ or from holomorphic curves in $\mathbb{C} P^{2}(4)$.

The paper is organized as follows. In Section 2, we recall the construction of the almost complex structure on $S^{6}(1)$, starting from the Cayley multiplication, as well as some basic facts about Lagrangian submanifolds. Next, we start our investigation of Lagrangian submanifolds of Type (vi). We show that, in contrast to the $\mathbb{C}^{3}$-case studied by Bryant [3], there does not exist any Lagrangian submanifold of Type (vi). As a corollary we remark that every Lagrangian submanifold of Type (k) which has constant scalar curvature must be equivariant and thus congruent to one of the 5 immersions of $S U(2)$ into $S^{6}(1)$, first described by Mashimo in [16]. This gives evidence to the following conjecture:

Conjecture 1. Let $\psi: M^{3} \rightarrow S^{6}(1)$ be a Lagrangian immersion with constant scalar curvature. Then $\psi$ is congruent with an open part of one of the 5 previously mentioned equivariant immersions.

The above conjecture can be seen as the analog for Lagrangian submanifolds of the well-known conjecture by Chern which states that the set of all possible values for the scalar curvature of a compact minimal hypersurface in a sphere is a discrete set. 
As a Lagrangian submanifold $M$ of $S^{6}(1)$ is always 3-dimensional, and thus odd-dimensional, it is a natural question to ask whether $M$ admits a Sasakian structure compatible with the induced metric. This problem was first considered in [12], where some partial results were obtained. In Section 4, we completely classify those Lagrangian submanifolds of $S^{6}(1)$ which admit such a Sasakian structure.

2. Preliminaries. We give a brief exposition of how the standard nearly Kähler structure on $S^{6}(1)$ arises in a natural manner from the Cayley multiplication. We also describe how we can use the vector cross product on $\mathbb{R}^{7}$ in order to define the Sasakian structure on $S^{5}(1)$. For further details about the Cayley numbers and their automorphism group $G_{2}$, we refer the reader to [18] and [15].

The multiplication on the Cayley numbers $\mathcal{O}$ may be used to define a vector cross product $\times$ on the purely imaginary Cayley numbers $\mathbb{R}^{7}$ using the formula

$$
u \times v=\frac{1}{2}(u v-v u),
$$

while the standard inner product on $\mathbb{R}^{7}$ is given by

$$
(u, v)=-\frac{1}{2}(u v+v u)
$$

It is now elementary [15] to show that

$$
u \times(v \times w)+(u \times v) \times w=2(u, w) v-(u, v) w-(w, v) u
$$

and that the triple scalar product $(u \times v, w)$ is skew symmetric in $u, v, w$.

Conversely, Cayley multiplication on $\mathcal{O}$ is given in terms of the vector cross product and the inner product by

$$
(r+u)(s+v)=r s-(u, v)+r v+s u+(u \times v), \quad r, s \in \operatorname{Re}(\mathcal{O}), u, v \in \operatorname{Im}(\mathcal{O})
$$

In view of (1), (2) and (4), it is clear that the group $G_{2}$ of automorphisms of $\mathcal{O}$ is precisely the group of isometries of $\mathbb{R}^{7}$ preserving the vector cross product.

An ordered basis $u_{1}, \ldots, u_{7}$ is said to be a $G_{2}$-frame if

$$
u_{3}=u_{1} \times u_{2}, \quad u_{5}=u_{1} \times u_{4}, \quad u_{6}=u_{2} \times u_{4}, \quad u_{7}=u_{3} \times u_{4} .
$$

For example, the standard basis $e_{1}, \ldots, e_{7}$ of $\mathbb{R}^{7}$ is a $G_{2}$-frame. Moreover, if $u_{1}, u_{2}, u_{4}$ are mutually orthogonal unit vectors with $u_{4}$ orthogonal to $u_{1} \times u_{2}$, then $u_{1}, u_{2}, u_{4}$ determine a unique $G_{2}$-frame $u_{1}, \ldots, u_{7}$ and $\left(\mathbb{R}^{7}, \times\right)$ is generated by $u_{1}, u_{2}, u_{4}$ subject to the relations:

$$
u_{i} \times\left(u_{j} \times u_{k}\right)+\left(u_{i} \times u_{j}\right) \times u_{k}=2 \delta_{i k} u_{j}-\delta_{i j} u_{k}-\delta_{j k} u_{i}
$$


Therefore, for any $G_{2}$-frame, we have the following very useful multiplication table [18]:

\begin{tabular}{r|rrrrrrr}
$\mathrm{x}$ & $u_{1}$ & $u_{2}$ & $u_{3}$ & $u_{4}$ & $u_{5}$ & $u_{6}$ & $u_{7}$ \\
\hline$u_{1}$ & 0 & $u_{3}$ & $-u_{2}$ & $u_{5}$ & $-u_{4}$ & $-u_{7}$ & $u_{6}$ \\
$u_{2}$ & $-u_{3}$ & 0 & $u_{1}$ & $u_{6}$ & $u_{7}$ & $-u_{4}$ & $-u_{5}$ \\
$u_{3}$ & $u_{2}$ & $-u_{1}$ & 0 & $u_{7}$ & $-u_{6}$ & $u_{5}$ & $-u_{4}$ \\
$u_{4}$ & $-u_{5}$ & $-u_{6}$ & $-u_{7}$ & 0 & $u_{1}$ & $u_{2}$ & $u_{3}$ \\
$u_{5}$ & $u_{4}$ & $-u_{7}$ & $u_{6}$ & $-u_{1}$ & 0 & $-u_{3}$ & $u_{2}$ \\
$u_{6}$ & $u_{7}$ & $u_{4}$ & $-u_{5}$ & $-u_{2}$ & $u_{3}$ & 0 & $-u_{1}$ \\
$u_{7}$ & $-u_{6}$ & $u_{5}$ & $u_{4}$ & $-u_{3}$ & $-u_{2}$ & $u_{1}$ & 0
\end{tabular}

The standard nearly Kähler structure on $S^{6}(1)$ is then obtained as follows:

$$
J u=x \times u, \quad u \in T_{x} S^{6}(1), \quad x \in S^{6}(1) .
$$

It is clear that $J$ is an orthogonal almost complex structure on $S^{6}(1)$. In fact $J$ is a nearly Kähler structure in the sense that the $(2,1)$-tensor field $G$ on $S^{6}(1)$ defined by

$$
G(X, Y)=\left(\tilde{\nabla}_{X} J\right) Y
$$

where $\tilde{\nabla}$ is the Levi-Civita connection on $S^{6}(1)$ is skew-symmetric. A straightforward computation also shows that

$$
G(X, Y)=X \times Y-\langle x \times X, Y\rangle x .
$$

For more information on the properties of ., $J$ and $G$, we refer to [2] and [10].

Let $M$ be a submanifold of $S^{6}(1)$. Then, $M$ is called Lagrangian provided that $J$ interchanges at every point $p$ of $M$ the tangent and the normal space. It is immediately clear that a Lagrangian submanifold is 3-dimensional. It was also shown by Ejiri, [13] that $M$ is minimal, orientable and that for tangent vector fields $X$ and $Y$ to $M$, $G(X, Y)$ is normal to $M$. Decomposing $\tilde{\nabla}_{X} Y$ and $\tilde{\nabla}_{X} J Y$ into a tangential and a normal component it follows that

$$
\nabla_{X}^{\perp} J Y=J \nabla_{X} Y+G(X, Y)
$$

and that the form defined by

$$
C(X, Y, Z)=\langle h(X, Y), J Z\rangle,
$$

is symmetric in $X, Y$ and $Z$.

Now, we finish this section, by recalling some basic facts about the Hopf lift, which we will need in some of the later sections. It is well-known (see for instance [1], page 32 or [11]) that the complex structure of $\mathbb{C}^{3}$ induces a Sasakian structure $(\varphi, \xi, \eta, g)$ on $S^{5}(1)$ starting from $\mathbb{C}^{3}$. This structure can also be expressed using the vector cross product. We consider $S^{5}(1)$ as the hypersphere in $S^{6}(1) \subset \mathbb{R}^{7}$ given by the equation $x_{4}=0$ and define:

$$
j: S^{5}(1) \rightarrow \mathbb{C}^{3}:\left(x_{1}, x_{2}, x_{3}, 0, x_{5}, x_{6}, x_{7}\right) \mapsto\left(x_{1}+i x_{5}, x_{2}+i x_{6}, x_{3}+i x_{7}\right) .
$$


Then at a point $p=\left(x_{1}, x_{2}, x_{3}, 0, x_{5}, x_{6}, x_{7}\right)$, the structure vector field $\xi$ is given by

$$
\xi(p)=\left(x_{5}, x_{6}, x_{7}, 0,-x_{1},-x_{2},-x_{3}\right)=e_{4} \times p,
$$

and for any tangent vector $v$, we get that

$$
\varphi(v)=v \times e_{4}-\left\langle v \times e_{4}, p\right\rangle p .
$$

Following [19], we call a submanifold $M^{n}$ of $S^{5}(1)$ invariant if $\varphi\left(T_{p} M\right) \subset T_{p} M$ for every $p$. If $n$ is odd, then $\xi$ is automatically tangent to $M$. Assume $n=3$. The Hopf fibration $h: S^{5}(1) \rightarrow \mathbb{C} P^{2}(4)$ annihilates $\xi$, i.e. $d h(\xi)=0$. Then if $M^{3}$ is invariant, $h\left(M^{3}\right)$ is a holomorphic curve. Conversely, let $\phi: N_{1} \rightarrow \mathbb{C} P^{2}(4)$ be a holomorphic curve, let $P N_{1}$ be the circle bundle over $N_{1}$ induced by the Hopf fibration and let $\psi$ be the immersion such that the following diagram commutes:

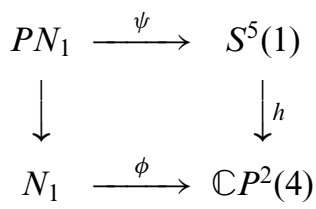

Then $\psi$ is an invariant immersion in the Sasakian space form $S^{5}(1)$ with structure vector field $\xi$ tangent along $\psi$.

3. Lagrangian submanifolds of Type (vi). In this section, we assume that $M$ is a Lagrangian submanifold of Type (vi). It is easy to see that this implies that at each point $p$ there exists an orthonormal basis $\left\{e_{1}, e_{2}, e_{3}\right\}$ such that

$$
\begin{array}{lll}
h\left(e_{1}, e_{1}\right)=\lambda_{1} J e_{1}, & h\left(e_{1}, e_{2}\right)=\lambda_{2} J e_{2}, & h\left(e_{3}, e_{1}\right)=\lambda_{3} J e_{3}, \\
h\left(e_{2}, e_{2}\right)=\lambda_{2} J e_{1}, & h\left(e_{2}, e_{3}\right)=0, & h\left(e_{3}, e_{3}\right)=\lambda_{3} J e_{1},
\end{array}
$$

where $0<\lambda_{1}=-\lambda_{2}-\lambda_{3}$ and $0 \neq \lambda_{2} \neq \lambda_{3} \neq 0$.

By a straightforward computation we obtain the following:

LEMMA 1. Let $\left\{e_{1}, e_{2}, e_{3}\right\}$ be the orthonormal basis defined previously. Then it follows that

$$
\begin{array}{lll}
\operatorname{Ric}\left(e_{1}, e_{1}\right)=1-\lambda_{2}^{2}-\lambda_{3}^{2}-\lambda_{2} \lambda_{3}, & \operatorname{Ric}\left(e_{1}, e_{2}\right)=0, & \operatorname{Ric}\left(e_{3}, e_{1}\right)=0, \\
\operatorname{Ric}\left(e_{2}, e_{2}\right)=1-\lambda_{2}^{2}, & \operatorname{Ric}\left(e_{2}, e_{3}\right)=0, & \operatorname{Ric}\left(e_{3}, e_{3}\right)=1-\lambda_{3}^{2} .
\end{array}
$$

Using the various conditions on $\lambda_{2}$ and $\lambda_{3}$, we see that the 1-1 symmetric tensor field $P$ associated with the Ricci tensor has at each point three different eigenvalues, all with multiplicity 1 . Hence there exist orthonormal vector fields $\left\{E_{1}, E_{2}, E_{3}\right\}$ defined on a neighborhood of the point $p$ and a non vanishing differentiable functions $\lambda_{2}, \lambda_{3}$, with $\lambda_{2}+\lambda_{3}<0$ and $\lambda_{2}-\lambda_{3} \neq 0$ such that

$$
\begin{array}{lll}
h\left(E_{1}, E_{1}\right)=-\left(\lambda_{2}+\lambda_{3}\right) J E_{1}, & h\left(E_{1}, E_{2}\right)=\lambda_{2} J E_{2}, & h\left(E_{3}, E_{1}\right)=\lambda_{3} J E_{3}, \\
h\left(E_{2}, E_{2}\right)=\lambda_{2} J E_{1}, & h\left(E_{2}, E_{3}\right)=0, & h\left(E_{3}, E_{3}\right)=\lambda_{3} J E_{1} .
\end{array}
$$


As $G\left(E_{1}, E_{2}\right)= \pm J E_{3}$, we may, by replacing $E_{3}$ with $-E_{3}$ if necessary, assume that $G\left(E_{1}, E_{2}\right)=J E_{3}$. We then introduce local functions $a_{1}, \ldots, c_{3}$ by

$$
\begin{array}{lll}
\nabla_{E_{1}} E_{1}=a_{1} E_{2}+a_{2} E_{3}, & \nabla_{E_{1}} E_{2}=-a_{1} E_{1}+a_{3} E_{3}, & \nabla_{E_{1}} E_{3}=-a_{2} E_{1}-a_{3} E_{2}, \\
\nabla_{E_{2}} E_{1}=b_{1} E_{2}+b_{2} E_{3}, & \nabla_{E_{2}} E_{2}=-b_{1} E_{1}+b_{3} E_{3}, & \nabla_{E_{2}} E_{3}=-b_{2} E_{1}-b_{3} E_{2}, \\
\nabla_{E_{3}} E_{1}=c_{1} E_{2}+c_{2} E_{3}, & \nabla_{E_{3}} E_{2}=-c_{1} E_{1}+c_{3} E_{3}, & \nabla_{E_{3}} E_{3}=-c_{2} E_{1}-c_{3} E_{2},
\end{array}
$$

Computing now all components of the Gauss equation, it then follows by a long but straightforward computation that the functions $\lambda_{2}, \lambda_{3}, a_{1}, \ldots, c_{3}$ have to satisfy the following system of partial differential equations:

$$
\begin{aligned}
& E_{2}\left(a_{1}\right)-E_{1}\left(b_{1}\right)=1-2 \lambda_{2}^{2}-\lambda_{2} \lambda_{3}+a_{1}^{2}+b_{1}^{2}+b_{2} c_{1}-b_{2} a_{3}+a_{2} b_{3}-a_{3} c_{1}, \\
& E_{3}\left(a_{2}\right)-E_{1}\left(c_{2}\right)=1-2 \lambda_{3}^{2}-\lambda_{2} \lambda_{3}+a_{2}^{2}+c_{2}^{2}+b_{2} c_{1}+b_{2} a_{3}-a_{1} c_{3}+a_{3} c_{1}, \\
& E_{2}\left(a_{2}\right)-E_{1}\left(b_{2}\right)=b_{1} a_{3}+b_{1} b_{2}+a_{1} a_{2}-a_{1} b_{3}+c_{2} b_{2}-c_{2} a_{3}, \\
& E_{3}\left(a_{1}\right)-E_{1}\left(c_{1}\right)=a_{2} c_{3}-a_{3} c_{2}+a_{1} a_{2}+b_{1} c_{1}+c_{1} c_{2}+b_{1} a_{3}, \\
& E_{2}\left(c_{2}\right)-E_{3}\left(b_{2}\right)=b_{1} c_{3}-b_{3} c_{1}-a_{2} b_{2}+a_{2} c_{1}-b_{2} b_{3}-c_{2} c_{3}, \\
& E_{2}\left(c_{1}\right)-E_{3}\left(b_{1}\right)=b_{3} c_{2}-c_{3} b_{2}+a_{1} c_{1}-a_{1} b_{2}-c_{1} c_{3}-b_{1} b_{3}, \\
& E_{3}\left(a_{3}\right)-E_{1}\left(c_{3}\right)=a_{1} c_{2}-a_{2} c_{1}+a_{2} a_{3}+a_{3} b_{3}+b_{3} c_{1}+c_{2} c_{3}, \\
& E_{1}\left(b_{3}\right)-E_{2}\left(a_{3}\right)=b_{1} a_{2}-a_{1} b_{2}-a_{1} a_{3}-b_{1} b_{3}+a_{3} c_{3}-c_{3} b_{2}, \\
& E_{3}\left(b_{3}\right)-E_{2}\left(c_{3}\right)=1+\lambda_{2} \lambda_{3}+b_{3}^{2}+c_{3}^{2}+a_{3} b_{2}-a_{3} c_{1}+b_{1} c_{2}-b_{2} c_{1} .
\end{aligned}
$$

The number of unknowns in the above equations can be reduced using the Codazzi equation which states that $(\nabla h)(X, Y, Z)=\left(\nabla_{X} h\right)(Y, Z)=\nabla_{X}^{\frac{1}{X}} h(Y, Z)-h\left(\nabla_{X} Y, Z\right)-$ $h\left(Y, \nabla_{X} Z\right)$ is totally symmetric in $X, Y$ and $Z$. In particular we obtain that

LEMMA 2. Let $\left\{E_{1}, E_{2}, E_{3}\right\}$ be the local orthonormal basis defined previously. Then, we have that there exists a function $c$ such that

$$
\begin{aligned}
& b_{2}=-c_{1}=-a_{3}=\frac{1}{4}, \\
& a_{2}=\left(1-\frac{\lambda_{3}}{\lambda_{2}}\right) b_{3}, \\
& a_{1}=\left(\frac{\lambda_{2}}{\lambda_{3}}-1\right) c_{3}, \\
& c_{2}=c \lambda_{3}, \\
& b_{1}=c \lambda_{2} .
\end{aligned}
$$

Moreover, the functions $\lambda_{2}$ and $\lambda_{3}$ satisfy the following system of differential equations:

$$
\begin{aligned}
& E_{1}\left(\lambda_{2}\right)=-c \lambda_{2}\left(3 \lambda_{2}+\lambda_{3}\right), \\
& E_{2}\left(\lambda_{2}\right)=3 c_{3} \lambda_{2}\left(\frac{\lambda_{2}}{\lambda_{3}}-1\right), \\
& E_{3}\left(\lambda_{2}\right)=b_{3}\left(\lambda_{2}-\lambda_{3}\right), \\
& E_{1}\left(\lambda_{3}\right)=-c \lambda_{3}\left(3 \lambda_{3}+\lambda_{2}\right), \\
& E_{2}\left(\lambda_{3}\right)=c_{3}\left(\lambda_{2}-\lambda_{3}\right), \\
& E_{3}\left(\lambda_{3}\right)=3 b_{3} \lambda_{3}\left(1-\frac{\lambda_{3}}{\lambda_{2}}\right) .
\end{aligned}
$$

Proof. As,

$$
\left(\nabla_{E_{2}} h\right)\left(E_{3}, E_{3}\right)=E_{2}\left(\lambda_{3}\right) J E_{1}+b_{1} \lambda_{3} J E_{2}+\left(3 b_{2}-1\right) \lambda_{3} J E_{3},
$$


and

$$
\left(\nabla_{E_{3}} h\right)\left(E_{2}, E_{3}\right)=c_{3}\left(\lambda_{2}-\lambda_{3}\right) J E_{1}+c_{2} \lambda_{2} J E_{2}+c_{1} \lambda_{3} J E_{3},
$$

it follows from the Codazzi equation $\left(\nabla_{E_{2}} h\right)\left(E_{3}, E_{3}\right)=\left(\nabla_{E_{3}} h\right)\left(E_{2}, E_{3}\right)$ that

$$
\begin{aligned}
c_{1} & =3 b_{2}-1, \\
\lambda_{3} b_{1} & =c_{2} \lambda_{2}, \\
E_{2}\left(\lambda_{3}\right) & =b_{3}\left(\lambda_{2}-\lambda_{3}\right) .
\end{aligned}
$$

Similarly, we obtain from $\left(\nabla_{E_{2}} h\right)\left(E_{3}, E_{2}\right)=\left(\nabla_{E_{3}} h\right)\left(E_{2}, E_{2}\right)$ that

$$
\begin{aligned}
b_{2} & =3 c_{1}+1, \\
E_{3}\left(\lambda_{2}\right) & =b_{3}\left(\lambda_{2}-\lambda_{3}\right) .
\end{aligned}
$$

Combining (16) and (19) it then follows that $c_{1}=-\frac{1}{4}$ and $b_{2}=\frac{1}{4}$. The remaining equations follow similarly from the other Codazzi equations.

Using the previous lemma, the differential equations given by (9) to (15) now imply that

$$
\begin{aligned}
E_{2}(c) & =\frac{1}{2} b_{3}\left(\frac{1}{\lambda_{2}}-\frac{1}{\lambda_{3}}\right), \\
E_{3}(c) & =\frac{1}{2} c_{3}\left(\frac{1}{\lambda_{3}}-\frac{1}{\lambda_{2}}\right), \\
E_{1}\left(c_{3}\right) & =-c c_{3} \lambda_{2}+\frac{1}{2} b_{3}, \\
E_{1}\left(b_{3}\right) & =-b_{3} c \lambda_{3}-\frac{1}{2} c_{3}, \\
E_{3}\left(c_{3}\right) & =3 b_{3} c_{3}-\frac{1}{2} c \frac{\lambda_{2} \lambda_{3}}{\lambda_{2}-\lambda_{3}}, \\
E_{2}\left(b_{3}\right) & =-3 b 3 c_{3}+\frac{1}{2} c \frac{\lambda_{2} \lambda_{3}}{\lambda_{2}-\lambda_{3}} .
\end{aligned}
$$

We now compute some integrability conditions. As $\nabla$ is torsion free, we know that for any function $f$, the following equations are satisfied:

$$
\begin{aligned}
& 0=E_{1}\left(E_{2}(f)\right)-E_{2}\left(E_{1}(f)\right)-\left(\nabla_{E_{1}} E_{2}\right)(f)+\left(\nabla_{E_{2}} E_{1}\right)(f), \\
& 0=E_{1}\left(E_{3}(f)\right)-E_{3}\left(E_{1}(f)\right)-\left(\nabla_{E_{1}} E_{3}\right)(f)+\left(\nabla_{E_{3}} E_{1}\right)(f), \\
& 0=E_{2}\left(E_{3}(f)\right)-E_{3}\left(E_{2}(f)\right)-\left(\nabla_{E_{2}} E_{3}\right)(f)+\left(\nabla_{E_{3}} E_{2}\right)(f) .
\end{aligned}
$$

It is straightforward to check that applying the above principle for the functions $\lambda_{2}$ and $\lambda_{3}$ does not yield any new equations. However, applying the first principle for the function $b_{3}$ yields the following differential equation:

$\lambda_{2} \lambda_{3}\left(\lambda_{2}-\lambda_{3}\right)\left(E_{3}\left(b_{3}\right)+E_{2}\left(c_{3}\right)\right)+\lambda_{2}^{2} \lambda_{3}^{2} E_{1}(c)=\left(\lambda_{2}-\lambda_{3}\right)\left(c_{3}^{2} \lambda_{2}\left(\lambda_{2}-4 \lambda_{3}\right)+b_{3}^{2} \lambda_{3}\left(4 \lambda_{2}-\lambda_{3}\right)\right)$. 
Combining this equation, together with the remaining Gauss equations, we then obtain that

$$
\begin{aligned}
E_{3}\left(b_{3}\right) & =\frac{\left(-5-32 b_{3}^{2}+16 c_{3}^{2}\right) \lambda_{3}+2 \lambda_{2}\left(5+16 b_{3}^{2}-8 c_{3}^{2}-8 \lambda_{3}^{2}-8 c^{2} \lambda_{3}^{2}\right)}{16\left(\lambda_{2}-\lambda_{3}\right)}, \\
E_{2}\left(c_{3}\right) & =\frac{\left(-5-32 c_{3}^{2}+16 b_{3}^{2}\right) \lambda_{2}+2 \lambda_{3}\left(5+16 c_{3}^{2}-8 b_{3}^{2}-8 \lambda_{2}^{2}-8 c^{2} \lambda_{2}^{2}\right)}{16\left(\lambda_{2}-\lambda_{3}\right)}, \\
E_{1}(c) & =\frac{-5-32 b_{3}^{2}+16 c_{3}^{2}}{16 \lambda_{2}}+\lambda_{2}\left(1+c^{2}+\frac{c_{3}^{2}}{\lambda_{3}^{2}}\right)+\frac{-5-32 c_{3}^{2}+16 b_{3}^{2}}{16 \lambda_{3}}+\lambda_{3}\left(1+c^{2}+\frac{b_{3}^{2}}{\lambda_{2}^{2}}\right) .
\end{aligned}
$$

Checking now the integrability conditions for $c, c_{3}$ and $b_{3}$ it immediately follows that $c=c_{3}=b_{3}=0$. Substituting these values in to the Gauss equations it follows that $\lambda_{2}=-\lambda_{3}$ which is a contradiction. Therefore, we have shown the following theorem:

THEOREM 2. There does not exist a Lagrangian submanifold of Type (vi) in $S^{6}(1)$.

4. Lagrangian submanifolds admitting a Sasakian structure. Throughout this section, we will assume that $M$ is a Lagrangian submanifold of $S^{6}(1)$ which admits a Sasakian structure which is compatible with the induced metric $\langle.,$.$\rangle on M$. This implies, see [1], that there exists a unit-length vector field $\xi$ on $M$, a 1-form $\eta$ and an endomorphism $\Phi$ satisfying:

$$
\begin{aligned}
\eta(X) & =\langle X, \xi\rangle, \\
\Phi^{2} & =-I+\eta \otimes \xi, \\
\Phi(\xi) & =0, \\
\eta \circ \Phi & =0, \\
\langle\Phi X, \Phi Y\rangle & =\langle X, Y\rangle-\eta(X) \eta(Y), \\
\left(\nabla_{X} \phi\right) Y & =\eta(Y) X-\langle X, Y\rangle \xi .
\end{aligned}
$$

Moreover, it is well known, see [1], that the above equations imply that

$$
\begin{aligned}
\nabla_{X} \xi & =-\Phi X \\
R(X, Y) \xi & =\langle\xi, Y\rangle X-\langle X, \xi\rangle Y .
\end{aligned}
$$

From [1], we recall the following theorem that the previous equation together with the fact that $\xi$ is a unit-length Killing vector field are the principal criteria for determining whether an odd-dimensional manifold admits a Sasakian structure compatible with a given metric.

LEMma 3. Let $M^{3}$ be a Riemannian manifold admitting a unit length Killing vector field $\xi$ such that

$$
R(X, Y) \xi=\langle\xi, Y\rangle X-\langle\xi, X\rangle Y
$$

Then $M$ admits a Sasakian structure which is compatible with the given metric. 
Taking now an orthonormal basis $\left\{e_{1}, e_{2}, e_{3}\right\}$ at a point $p$ of $M^{3}$ such that $e_{3}=\xi$, it immediately follows from (22) that

$$
\begin{array}{lll}
\operatorname{Ric}\left(e_{1}, e_{1}\right)=\frac{1}{2}\left(\left\langle R\left(e_{1}, e_{2}\right) e_{2}, e_{1}\right\rangle+1\right), & \operatorname{Ric}\left(e_{1}, e_{2}\right)=0, & \operatorname{Ric}\left(e_{3}, e_{1}\right)=0, \\
\operatorname{Ric}\left(e_{2}, e_{2}\right)=\frac{1}{2}\left(\left\langle R\left(e_{1}, e_{2}\right) e_{2}, e_{1}\right\rangle+1\right), & \operatorname{Ric}\left(e_{2}, e_{3}\right)=0, & \operatorname{Ric}\left(e_{3}, e_{3}\right)=1 .
\end{array}
$$

This implies that all sectional curvatures at the point $p$ equal 1 or the associated endomorphism $P$ has two eigenvalues: one with multiplicity 1 and one with multiplicity 2. Moreover, in the second case, the eigenvalue with multiplicity 1 equals 1 and the vector field $\xi$ spans the corresponding 1-dimensional eigenspace. We now recall the following lemma from [9] about quasi-Einstein submanifolds.

LEMMA 4. Let $M^{3}$ be a 3-dimensional Lagrangian submanifold of $S^{6}$ with the second fundamental form $h$. Then the Ricci endomorphism P, associated with the Ricci tensor Ric, satisfies:

(i) 1 is an eigenvalue of $P$,

(ii) $P$ has an eigenvalue with multiplicity at least 2 ,

if and only if $p$ is a totally geodesic point or there exist a orthonormal basis $\left\{e_{1}, e_{2}, e_{3}\right\}$ of $T_{p} M$ such that either

$$
\begin{array}{ll}
h\left(e_{1}, e_{1}\right)=\lambda J e_{1}, & h\left(e_{2}, e_{2}\right)=-\lambda J e_{1}, \\
h\left(e_{1}, e_{2}\right)=-\lambda J e_{2}, & h\left(e_{2}, e_{3}\right)=0, \\
h\left(e_{1}, e_{3}\right)=0, & h\left(e_{3}, e_{3}\right)=0,
\end{array}
$$

where $\lambda$ is a non-zero number. Moreover, in the second case, the 1-dimensional eigenspace is determined by $e_{3}$.

It follows immediately from the above lemma that a Lagrangian submanifold which admits a Sasakian structure satisfies Chen's equality. Moreover, if necessary by restricting to an open dense subset, we may assume that either $M$ is totally geodesic or in a neighborhood of any point $p$ of $M^{3}$ there exist an orthonormal basis $\left\{E_{1}, E_{2}, E_{3}\right\}$ such that

$$
\begin{array}{lll}
h\left(E_{1}, E_{1}\right)=\lambda J E_{1}, & h\left(E_{2}, E_{2}\right)=-\lambda J E_{1}, & h\left(E_{1}, E_{2}\right)=-\lambda J E_{2}, \\
h\left(E_{2}, E_{3}\right)=0, & h\left(E_{1}, E_{3}\right)=0, & h\left(E_{3}, E_{3}\right)=0,
\end{array}
$$

where $E_{3}=\xi$. Now, we proceed as follows. We take the frame constructed in the previous lemma. As $G\left(E_{3}, E_{1}\right)$ is a normal vector which is orthogonal to both $J E_{3}$ and $J E_{1}$ it follows that $G\left(E_{3}, E_{1}\right)= \pm E_{2}$. Therefore, if necessary by changing the sign of $E_{2}$, we may assume that $G\left(E_{3}, E_{1}\right)=J E_{2}$. It then follows that $G\left(E_{1}, E_{2}\right)=J E_{3}$ and $G\left(E_{2}, E_{3}\right)=J E_{1}$. Moreover, we also have that $\Phi E_{1}=\epsilon E_{2}$, where $\epsilon= \pm 1$. It then follows from (21) that

$$
\begin{aligned}
& \nabla_{E_{1}} E_{3}=-\epsilon E_{2}, \\
& \nabla_{E_{2}} E_{3}=\epsilon E_{1}, \\
& \nabla_{E_{3}} E_{3}=0 .
\end{aligned}
$$


Introducing now functions $a, b$ and $c$, it follows that we can express the other components of the connection $\nabla$ respectively by

$$
\begin{array}{ll}
\nabla_{E_{1}} E_{1}=c E_{2}, & \nabla_{E_{1}} E_{2}=-c E_{1}+\epsilon E_{3}, \\
\nabla_{E_{2}} E_{1}=d E_{2}-\epsilon E_{3}, & \nabla_{E_{2}} E_{2}=-d E_{1}, \\
\nabla_{E_{3}} E_{1}=f E_{2}, & \nabla_{E_{3}} E_{2}=-f E_{1} .
\end{array}
$$

Using now the Codazzi equation, see also Lemma 5.3 of [8], it follows that $f=$ $-\frac{1}{3}(1+\epsilon)$. We now consider two different cases. First, we assume that $\epsilon=-1$. In that case, we have that

$$
\begin{aligned}
\tilde{\nabla}_{E_{1}} J E_{3} & =G\left(E_{1}, E_{3}\right)+J \tilde{\nabla}_{E_{1}} E_{3} \\
& =-J E_{2}+J E_{2}+h\left(E_{1}, E_{3}\right)=0, \\
\tilde{\nabla}_{E_{2}} J E_{3} & =G\left(E_{2}, E_{3}\right)+J \tilde{\nabla}_{E_{2}} E_{3} \\
& =J E_{1}-J E_{1}+h\left(E_{2}, E_{3}\right)=0, \\
\tilde{\nabla}_{E_{3}} J E_{3} & =J \tilde{\nabla}_{E_{3}} E_{3}=0 .
\end{aligned}
$$

Hence, $J E_{3}$ is a constant vector along $M$ which is tangent to $S^{6}(1)$. Consequently $M$ lies in the totally geodesic $S^{5}(1)$ which is obtained as the intersection of $S^{6}(1)$ with the linear hyperplane orthogonal to $J E_{3}$. Using now the classification of Lagrangian submanifolds contained in a totally geodesic subspace, see Theorems 1 and 4 of [11], we obtain that $M$ is locally congruent to the Hopf lift of a complex curve in $\mathbb{C} P^{2}(4)$ to $S^{5}(1)$ and $S^{5}(1)$ is immersed in $S^{6}(1)$ as described in Section 2. As the Hopf lift of a complex curve is a Sasakian manifold, the converse is obvious.

Finally, we consider the case that $\epsilon=1$. In this case, we denote the immersion by $F$ and proceed as in [11] to show that the map $J E_{3}=F \times E_{3}$ defines an almost complex curve. Specializing the formulas there, to our case, i.e. using that the functions $a$ and $b$ defined in [11] are respectively given by $a=0$ and $b=1$, we get that:

$$
\begin{aligned}
D_{E_{1}}\left(F \times E_{3}\right) & =2 E_{1} \times E_{3}, \\
D_{E_{2}}\left(F \times E_{3}\right) & =2 E_{2} \times E_{3}, \\
D_{E_{3}}\left(F \times E_{3}\right) & =0, \\
D_{E_{1}}\left(E_{1} \times E_{3}\right) & =\left(c E_{2}+\lambda F \times E_{1}-F\right) \times E_{3}-E_{1} \times E_{2}=c E_{2} \times E_{3}-\lambda E_{2}-2 F \times E_{3}, \\
D_{E_{2}}\left(E_{1} \times E_{3}\right) & =\left(d E_{2}-\lambda F \times E_{2}\right) \times E_{3}=d E_{2} \times E_{3}-\lambda E_{1}, \\
D_{E_{1}}\left(E_{2} \times E_{3}\right) & =\left(-c E_{1}-\lambda F \times E_{2}\right) \times E_{3}=-c E_{1} \times E_{3}-\lambda E_{1}, \\
D_{E_{2}}\left(E_{2} \times E_{3}\right) & =\left(-d E_{1}-\lambda F \times E_{1}-F\right) \times E_{3}+E_{2} \times E_{1}=-d E_{1} \times E_{3}+\lambda E_{2}-2 F \times E_{3}, \\
D_{E_{1}} E_{1} & =c E_{2}+\lambda F \times E_{1}-F=c E_{2}+\lambda E_{2} \times E_{3}-F, \\
D_{E_{1}} E_{2} & =-c E_{1}-\lambda F \times E_{2}+E_{3}=-c E_{1}+\lambda E_{1} \times E_{3}+E_{3}, \\
D_{E_{2}} E_{1} & =d E_{2}-\lambda F \times E_{2}-E_{3}=d E_{2}+\lambda E_{1} \times E_{3}-E_{3}, \\
D_{E_{2}} E_{2} & =-d E_{1}-\lambda F \times E_{1}-F=-d E_{1}-\lambda E_{2} \times E_{3}-F .
\end{aligned}
$$

The above formulas now imply immediately that the complex curve defined by $J E_{3}$ is superminimal. A representation of superminimal complex curves was obtained in [4]. It now follows from [11] that $M$ is obtained as in Theorem 2 of [11], starting from a superminimal complex curve in $S^{6}(1)$. 
In order to show that we can start with an arbitrary superminimal complex curve it is sufficient to check that the vector field $\frac{\partial}{\partial t}$, defined in the proof of Theorem 2 of [11], is a Killing vector field satisfying the conditions of Lemma 3, which can be verified by a straightforward computation. Therefore, we have shown the following theorem:

THEOREM 3. Let $\psi: M^{3} \rightarrow S^{6}(1)$ be a Lagrangian immersion. Then $M$ admits a Sasakian structure compatible with the induced metric if and only if locally $M$ is congruent with

(i) a totally geodesic immersion,

(ii) the immersion $F: P N_{1} \rightarrow S^{6}(1)$, where $\phi: N_{1} \longrightarrow \mathbb{C} P^{2}(4)$ is a holomorphic curve in $\mathbb{C} P^{2}(4), P N_{1}$ is the circle bundle over $N_{1}$ induced by the Hopf fibration $p$ : $S^{5}(1) \rightarrow \mathbb{C} P^{2}(4), S^{5}(1)$ is embedded in $S^{6}$ as described in Section 2 and $F$ is the isometric immersion such that the following diagram commutes:

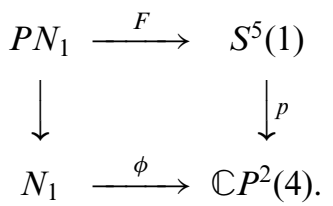

(iii) the immersion

$$
G: U N_{2} \rightarrow S^{6}(1): v \mapsto \bar{\phi}_{\star}(v) \times \frac{\alpha(v, v)}{\|\alpha(v, v)\|},
$$

where $\bar{\phi}: N_{2} \longrightarrow S^{6}(1)$ is a superminimal almost complex curve (with second fundamental form $\alpha$ ) without totally geodesic points and $U N_{2}$ is the unit tangent bundle over $\mathrm{N}_{2}$.

\section{REFERENCES}

1. D. E. Blair, Contact manifolds im Riemannian geometry, Lecture Notes in Mathematics No. 509 (Springer-Verlag, 1976).

2. J. Bolton, L. Vrancken and L. M. Woodward, On almost complex curves in the nearly Kaehler 6-sphere, Quart. J. Math. Oxford Ser (2) 45 (1994), 407-427.

3. R. L. Bryant, Second order families of special Lagrangian 3-folds, preprint.

4. R. L. Bryant, Submanifolds and special structures on the octonions, J. Differential Geometry 17 (1982), 185-232.

5. E. Calabi and H. Gluck, What are the best almost complex structures on the 6-sphere?, in Differential geometry: geometry in mathematical physics and related topics (Amer. Math. Soc., 1993), 99-106.

6. B. Y. Chen, Some pinching and classification theorems for minimal submanifolds, Archiv. Math. (Basel) 60 (1993), 568-578.

7. B. Y. Chen, F. Dillen, L. Verstraelen and L. Vrancken, Two equivariant totally real immersions into the nearly Kähler 6-sphere and their characterization, Japanese J. Math. (N.S) 21 (1995), 207-222.

8. B. Y. Chen, F. Dillen, L. Verstraelen and L. Vrancken, Characterizing a class of totally real submanifolds of $S^{6}(1)$ by their sectional curvatures, Tôhoku Math. J. 47 (1995), 185-198.

9. R. Deszcz, F. Dillen, L. Verstraelen and L. Vrancken, Quasi-Einstein totally real submanifolds of the nearly Kähler 6-sphere, Tôhoku Math. J. 51 (1999), 461-478.

10. F. Dillen, L. Verstraelen and L. Vrancken, On problems of U. Simon concerning minimal submanifolds of the nearly Kaehler 6-sphere, Bull. Amer. Math. Soc. 19 (1988), 433-438.

11. F. Dillen and L. Vrancken, Totally real submanifolds in $S^{6}(1)$ satisfying Chen's equality, Trans. Amer. Math. Soc. 348 (1996), 1633-1646. 
12. S. Deshmukh and K. El Hadi, A Sasakian structure on a 3-dimensional totally real submanifold of the nearly Kähler 6-sphere, Panamer. Math. J. 2 (1992), 43-52.

13. N. Ejiri, Totally real submanifolds in a 6-sphere, Proc. Amer. Math. Soc. 83 (1981), $759-763$

14. N. Ejiri, Equivariant minimal immersions of $S^{2}$ into $S^{2 m}$, Trans. Amer. Math. Soc. 297 (1986), 105-124.

15. R. Harvey and H. B. Lawson, Calibrated geometries, Acta Math. 148 (1982), 47-157.

16. K. Mashimo, Homogeneous totally real submanifolds of $S^{6}(1)$, Tsukuba J. Math. 9 (1985), 185-202.

17. B. Palmer, Calibrations and Lagrangian submanifolds in the six sphere, Tôhoku Math. J. 50 (1998), 303-315.

18. R. M. Wood, Framing the exceptional Lie group $G_{2}$, Topology 15 (1976), 303-320.

19. K. Yano and S. Ishihara, Invariant submanifolds of an almost contact manifold, Kodai. Math. Sem. Rep. 21 (1969), 350-364. 